\title{
Rat pial microvascular responses to transient bilateral common carotid artery occlusion and reperfusion: quercetin's mechanism of action
}

\author{
D. Lapi ${ }^{1}$, S. Vagnani ${ }^{2}$, G. Pignataro ${ }^{1}$, E. Esposito ${ }^{1}$, M. Paterni ${ }^{3}$ and A. Colantuoni ${ }^{1}$ \\ 'Department of Neuroscience, "Federico II" University Medical School, Naples, Italy \\ ${ }^{2}$ Rheumatology Unit, Department of Internal Medicine, University of Pisa, Pisa, Italy \\ ${ }^{3}$ National Research Council Institute of Clinical Physiology, Pisa, Italy
}

\section{Edited by:}

Gerald A. Meininger, Central Michigan

University, USA

Reviewed by:

Mohammad Imtiaz, The University of Calgary, Canada

Gregory Hare, University of Toronto,

Canada

D. Lapi, Department of

Neuroscience, "Federico II"

University Medical School, Via S.

Pansini, 5, 80121 Naples, Italy.

e-mail:d.lapi@dfb.unipi.it
*Correspondence:

The aim of the present study was to assess quercetin's mechanism of action in rat pial microvessels during transient bilateral common carotid artery occlusion (BCCAO) and reperfusion. Rat pial microcirculation was visualized using fluorescence microscopy through a closed cranial window. Pial arterioles were classified in five orders of branchings. In ischemic rats, 30 min BCCAO and 60 min reperfusion caused arteriolar diameter decrease, microvascular leakage, leukocyte adhesion in venules, and reduction of capillary perfusion. Quercetin highest dose determined dilation in all arteriolar orders, by $40 \pm 4 \%$ of baseline in order 2 vessels, and prevented microvascular permeability [0.15 \pm 0.02 normalized gray levels (NGL)], leukocyte adhesion, and capillary failure. Protein kinase $C(P K C)$ inhibition exerted by chelerythrine prior to quercetin attenuated quercetin-induced effects: order 2 arterioles dilated by $19.0 \pm 2.4 \%$ baseline, while there was an increase in permeability $(0.40 \pm 0.05 \mathrm{NGL})$ and leukocyte adhesion with a marked decrease in capillary perfusion. Tyrosine kinase (TK) inhibition by tyrphostin 47 prior to quercetin lessened smaller pial arterioles responses, dilating by $20.7 \pm 2.5 \%$ of baseline, while leakage increased $(0.39 \pm 0.04$ NGL) sustained by slight leukocyte adhesion and ameliorated capillary perfusion. Inhibition of endothelium nitric oxide synthase (eNOS) by $\mathrm{N}^{\mathrm{G}}$-nitro-L-arginine-methyl ester (L-NAME) prior to PKC or TK reduced the quercetin's effects on pial arteriolar diameter and leakage. eNOS inhibition by L-NAME reduced quercetin effects on pial arteriolar diameter and leakage. Finally, combined inhibition of PKC and TK prior to quercetin abolished quercetininduced effects, decreasing eNOS expression, while blocking ATP-sensitive potassium $\left(\mathrm{K}_{\text {ATP }}\right.$ ) channels by glibenclamide suppressed arteriolar dilation. In conclusion, the protective effects of quercetin could be due to different mechanisms resulting in NO release throughout PKC and TK intracellular signaling pathway activation.

Keywords: bilateral common carotid artery occlusion, reperfusion, pial microcirculation, quercetin, nitric oxide, protein kinase $C$, tyrosine kinase

\section{INTRODUCTION}

The cerebral transient hypoperfusion induced in rats by bilateral common carotid artery occlusion (BCCAO) and followed by reperfusion has been shown to induce a decrease in pial arteriolar diameter, disruption of the blood-brain barrier, leukocyte adhesion, and a reduction in capillary perfusion. These microvascular responses were significantly affected by quercetin, a natural flavonoid, effective in preventing arteriolar diameter decrease and macromolecular leakage, in reducing leukocyte adhesion, and facilitating capillary perfusion (Lapi et al., 2012). These protective quercetin effects have been suggested to be related to nitric oxide release, throughout endothelium nitric oxide synthase (eNOS) activation to increase as expression and activity detected by Western blotting (Lapi et al., 2012). Conversely, nitric oxide (NO) increase was not related to activation of neuronal nor inducible NOS. Moreover, the protection exerted by quercetin, especially on leukocyte adhesion, has been correlated to decrease in formation of reactive oxygen species (ROS), as reported in the joined paper (Huk et al., 1998; Rice-Evans, 2001; Lapi et al., 2012). However, several studies have suggested that quercetin can modulate vascular tone by protein kinase C (PKC) or cAMP- or cGMPphosphodiesterase or tyrosine kinase (TK) intracellular signaling pathways (Picq et al., 1989; Cogolludo et al., 2007; Negash et al., 2007; Chiwororo and Ojewole, 2010). To date, quercetin action mechanism remains partially unexplained.

According to our results in the rat pial microcirculation, we hypothesized pial arterioles dilation, prevention of leakage, and preservation of capillary perfusion during BCCAO and reperfusion may be due to activation of PKC or/and TK intracellular signaling pathway, with consequent increase in NO production and release. In several experimental models of preconditioning in different organs the protection is exerted through activation of PKC and TK signaling pathways (Fryer et al., 1999; Pagliaro et al., 2001; Sakamoto et al., 2005). The present study was aimed to 
investigate the quercetin's mechanism of action in rat pial microcirculation during BCCAO and reperfusion to gain new insight in prevention of ischemic injury.

We assessed the role of PKC and TK intracellular signaling pathways in the protection exerted by quercetin. We inhibited, indeed, PKC by chelerythrine or/and TK by tyrphostin 47; moreover, either inhibition was coupled to eNOS blockade by $\mathrm{N}^{\mathrm{G}}$ nitro-L-arginine-methyl ester (L-NAME) to clarify the quercetin's mechanism of action. Furthermore, potassium channels have been suggested to be activated during ischemia reperfusion injury and preconditioning in experimental models through $\mathrm{PKC}$ and $\mathrm{TK}$ signaling pathway stimulation (Pagliaro et al., 2001). Therefore, we assessed the role of potassium channels in the dilation induced by quercetin, blocking ATP-sensitive potassium $\left(\mathrm{K}_{\mathrm{ATP}}\right)$ channels by glibenclamide. Finally, we investigated the effects of combined PKC and TK inhibition on eNOS expression.

We used in vivo fluorescence technique to visualize rat pial microcirculation to determine changes in pial arteriole diameter, permeability increase, leukocyte adhesion, and capillary perfusion, as previously reported (Lapi et al., 2012). These data might be important to clarify the mechanisms effective in brain damage during hypoperfusion and reperfusion and to improve strategies against brain injury.

\section{MATERIALS AND METHODS EXPERIMENTAL GROUPS}

Male Wistar rats weighing 250-300 g (Harlan, Italy) were randomly assigned to eight group: (1) The first group was composed by the animals not subjected to BCCAO and reperfusion [ShamOperated (S) group, $n=14$ ], as indicated in the previous paper (Lapi et al., 2012). Moreover, sham-operated rats received intravenously (i.v.) chelerythrine, $3.0 \mathrm{mg} / \mathrm{Kg}$ b.w. (group SC, $n=5$ ) or i.v. tyrphostin $47,2.2 \mathrm{mg} / \mathrm{Kg}$ b.w. (group ST, $n=5$ ), or i.v. glibenclamide, $1.0 \mathrm{mg} / 100 \mathrm{~g}$ b.w. (group $\mathrm{SG}, n=5$ ). (2) Ischemic rats (group I, $n=20$ ) were treated with $1.5 \mathrm{ml}$ vehicle (physiological saline solution), i.v. injected, and subjected to $30 \mathrm{~min}$ of $\mathrm{BCCAO}$ and $60 \mathrm{~min}$ of reperfusion, as reported in the previous manuscript (Lapi et al., 2012). (3) The third group ( $Q_{3}$ group, $n=14$ ) was treated with i.v. quercetin $5.0 \mathrm{mg} / \mathrm{Kg}$ b.w. (4) The fourth group of rats (CQ group, $n=9$ ) received i.v. chelerythrine (3.0 mg/Kg b.w.) prior to i.v. quercetin ( $5.0 \mathrm{mg} / \mathrm{Kg}$ b.w.). (5) The fifth group of animals (group TQ, $n=9$ ) was subjected to i.v. treatment with tyrphostin $47(2.2 \mathrm{mg} / \mathrm{Kg}$ b.w.) before quercetin (5.0 mg/Kg b.w.). (6) The sixth group of rats (LCQ group, $n=9$ ) was treated with i.v. L-NAME (10.0 mg/Kg b.w.) before i.v. chelerythrine $(3.0 \mathrm{mg} / \mathrm{Kg}$ b.w.) and i.v. quercetin $(5.0 \mathrm{mg} / \mathrm{Kg}$ b.w.) with an interval of $10 \mathrm{~min}$ among each drug administration. (7) The seventh group of rats (group LTQ, $n=9$ ) was treated with i.v. L-NAME (10.0 mg/Kg b.w.) prior to i.v. tyrphostin $47(2.2 \mathrm{mg} / \mathrm{Kg}$ b.w.) and i.v. quercetin ( $5.0 \mathrm{mg} / \mathrm{Kg}$ b.w.) with an interval of $10 \mathrm{~min}$ after each drug administration. (8) The eighth group of animals (CTQ group, $n=14$ ) was treated with i.v. chelerythrine (3.0 mg/Kg b.w.) prior to i.v. tyrphostin 47 (2.2 mg/Kg b.w.) and i.v. quercetin $(5.0 \mathrm{mg} / \mathrm{Kg}$ b.w. $)$ with an interval of $10 \mathrm{~min}$ after each drug administration. Nine animals were utilized for microvascular studies, five to evaluate the eNOS expression by Western blotting. (9) The ninth group of rats (group GQ, $n=9$ ) was treated with i.v. glibenclamide $(1.0 \mathrm{mg} / 100 \mathrm{~g}$ b.w.) prior to quercetin $(5.0 \mathrm{mg} / \mathrm{Kg}$ b.w.). (10) Finally, the last group of rats of rats received chelerythrine (3.0 mg/Kg b.w.) or tyrphostin $47(2.2 \mathrm{mg} / \mathrm{Kg}$ b.w.) or glibenclamide (1.0 mg/100 g b.w.; group C, T, and G, $n=9$, respectively), i.v. injected 10 min before BCCAO and at the beginning of reperfusion.

\section{DRUGS ADMINISTRATION}

Quercetin solutions were obtained dissolving $5.0 \mathrm{mg} / \mathrm{Kg}$ b.w. in $0.5 \mathrm{ml}$ of saline solution and i.v. infused $(3 \mathrm{~min}$ ) to the rats $10 \mathrm{~min}$ before $\mathrm{BCCAO}$ and at the beginning of reperfusion (Cho et al., 2006).

Chelerythrine (3.0 mg/Kg b.w.; Colantuoni et al., 2005), tyrphostin 47 (2.2 mg/Kg b.w.), glibenclamide (1.0 mg/100 Kg b.w.), and L-NAME (10.0 mg/Kg b.w.; Xu et al., 2002; Lapi et al., 2008), were dissolved in $0.5 \mathrm{ml}$ of saline solution, respectively. Each substance was i.v. administered $10 \mathrm{~min}$ prior to quercetin $(5.0 \mathrm{mg} / \mathrm{Kg}$ b.w.) before BCCAO and at the beginning of reperfusion (Lapi et al., 2012). The drugs were purchased by Sigma Chemical, St. Louis, MO, USA.

In previous experiments i.v. infusion of $\mathrm{L}-\mathrm{NAME}$ at the dosage of $10 \mathrm{mg} / \mathrm{Kg}$ b.w., chosen for the present study, abolished vasodilation due to topical application of acetylcholine, $100 \mu \mathrm{M}(n=10)$, while the diameter increase was by $42.3 \pm 3.2 \%$ of baseline in sham-operated animals treated with acetylcholine, $n=10$ (Oriji, 1999).

\section{ANIMAL PREPARATION}

Anesthesia was induced with $\alpha$-chloralose (50 mg/Kg b.w., i.p.) plus urethane $(600 \mathrm{mg} / \mathrm{Kg}$ b.w., i.p.) and maintained with urethane alone $(100 \mathrm{mg} / \mathrm{Kg}$ b.w., i.v. every hour). Rats were tracheotomized, paralyzed with tubocurarine chloride $(1 \mathrm{mg} / \mathrm{Kg} \cdot \mathrm{h}$, i.v.) and mechanically ventilated with room air and supplemental oxygen, as previously reported (Lapi et al., 2012). Briefly, the right and left common carotid arteries were isolated for successive clamping. A catheter was placed in the left femoral artery, to measure arterial blood pressure, and in the right femoral vein for fluorescent tracers injection [fluorescein isothiocyanate bound to dextran, molecular weight $70 \mathrm{KDa}$ (FD 70), $50 \mathrm{mg} / 100 \mathrm{~g}$ b.w., i.v. as $5 \% \mathrm{wt} / \mathrm{vol}$ solution, and rhodamine $6 \mathrm{G}, 1 \mathrm{mg} / 100 \mathrm{~g}$ b.w. in $0.3 \mathrm{ml}$, as a bolus with supplemental injection throughout BCCAO and reperfusion (final volume $0.3 \mathrm{ml} \cdot 100 \mathrm{~g}^{-1} \cdot \mathrm{h}^{-1}$ ) to label leukocytes for adhesion evaluation] and of drugs. Blood gas measurements were carried out on arterial blood samples (ABL5; Radiometer, Copenhagen, Denmark). Throughout all experiments, mean arterial blood pressure, heart rate, respiratory $\mathrm{CO}_{2}$, and blood gases values were recorded and stable settled within physiological ranges. Rectal temperature was monitored and preserved at $37.0 \pm 0.5^{\circ} \mathrm{C}$, as previously reported (Lapi et al., 2012).

To observe the pial microcirculation, a closed cranial window $(4 \mathrm{~mm} \times 5 \mathrm{~mm})$ was implanted above the left frontoparietal cortex (posterior $1.5 \mathrm{~mm}$ to bregma; lateral, $3 \mathrm{~mm}$ to the midline; Chau et al., 2002). The dura mater was gently removed and a 150- $\mu \mathrm{m}-$ thick quarz microscope coverglass was sealed to the bone with dental cement. The brain parenchyma was continuously superfused with artificial cerebrospinal fluid (aCSF; Ngai et al., 1988; Moreau et al., 1995). The rate of superfusion was $0.5 \mathrm{ml} / \mathrm{min}$ 
controlled by a peristaltic pump. The composition of the aCSF was: $119.0 \mathrm{mM} \mathrm{NaCl}, 2.5 \mathrm{mM} \mathrm{KCl}, 1.3 \mathrm{mM} \mathrm{MgSO}_{4} \cdot 7 \mathrm{H}_{2} \mathrm{O}, 1.0 \mathrm{mM}$ $\mathrm{NaH}_{2} \mathrm{PO}_{4}, 26.2 \mathrm{mM} \mathrm{NaHCO}, 2.5 \mathrm{mM} \mathrm{CaCl}_{2}$, and $11.0 \mathrm{mM}$ glucose (equilibrated with $10.0 \% \mathrm{O}_{2}, 6.0 \% \mathrm{CO}_{2}$, and $84.0 \% \mathrm{~N}_{2} ; \mathrm{pH}$ $7.38 \pm 0.02$ ). The temperature was settled at $37.0 \pm 0.5^{\circ} \mathrm{C}$.

Cerebral blood flow reduction was produced by placement of two atraumatic microvascular clips for $30 \mathrm{~min}$ on common carotid arteries, previously isolated. After removing the clamp, the pial microcirculation was observed for $60 \mathrm{~min}$ (reperfusion period).

All experiments conform to the Guide for the Care and Use of Laboratory Animals published by the US National Institutes of Health (NIH Publication No. 85-23, revised 1996) and to institutional rules for the care and handling of experimental animals. The protocol was approved by the "Federico II" University of Naples Ethical Committee.

\section{INTRAVITAL MICROSCOPY AND MICROVASCULAR PARAMETER EVALUATION}

Observations of pial vessels were conducted by a fluorescence microscope (Leitz Orthoplan), as previously described (Lapi et al., 2012). Epiillumination was provided by a $100-\mathrm{W}$ mercury lamp using the appropriate filters for FITC, for rhodamine $6 \mathrm{G}$, and a heat filter (Leitz KG1). The pial microcirculation was televised with a DAGE MTI 300RC low-light level digital camera and recorded by a computer based frame grabber (Pinnacle DC 10 plus, Avid Technology, MA, USA).

Microvascular measurements were made off-line using a computer-assisted imaging software system (MIP Image, CNR Institute of Clinical Physiology, Pisa, Italy). We reported data under baseline conditions, at the end of BCCAO and at the end of reperfusion (RE). In Figure 1 we showed the time-dependent changes in arteriolar diameters to clarify the time-dependent pattern of order 2 arteriole response.

In each animal one order 4 arteriole, two order 3 , and two order 2 arterioles were studied during each experiment. We chose to present only the data regarding order 2 vessels, the most responsive arterioles, as previously reported (Lapi et al., 2012).

Arteriolar diameters were measured with a computer-assisted method (MIP Image, CNR, frame by frame). The results of diameter measurements were comparable with those obtained by shearing method $( \pm 0.5 \mu \mathrm{m})$. To avoid bias due to single operator measurements, two independent "blinded" operators measured the vessel diameters. Their measurements overlapped in all cases.

The increase in permeability was calculated and reported as normalized gray levels (NGL): NGL $=(I-\mathrm{Ir}) / \mathrm{Ir}$, where $\mathrm{Ir}$ is the average baseline gray level at the end of vessel filling with fluorescence (average of five windows located outside the blood vessels with the same windows being used throughout the experimental procedure), and $I$ is the same parameter at the end of BCCAO or RE.

Adherent leukocytes (i.e., cells on vessel walls that did not move over a 30-s observation period) were quantified in terms of number/100 $\mu \mathrm{m}$ of venular length (v.l.)/30 s using higher magnification $(32 \times$, microscope objectives). In each experimental group 45 venules were studied.

Perfused capillary length (PCL) was measured by MIP Image in an area of $150 \mu \mathrm{m} \times 150 \mu \mathrm{m}$. In this system the length of perfused

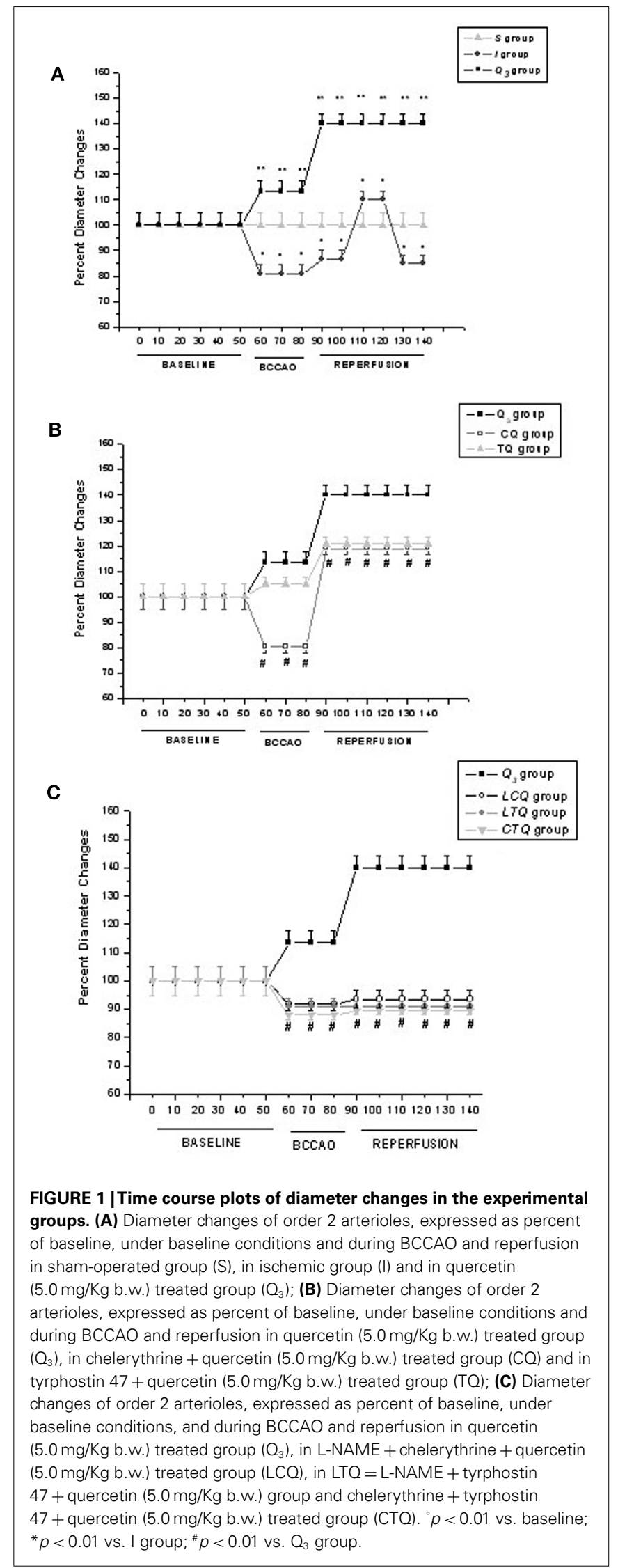


capillaries is easily established by the automated process because it is outlined by dextran (Xu et al., 2002).

Moreover, for each experimental group 27 pial venules were studied to evaluate the single pial venule blood flow, $\mathrm{Q}$, calculated according to the following equation: $\mathrm{Q}=\alpha \times V_{\mathrm{CL}} \times \mathrm{A}$, where $\alpha$ was a constant related to the vessel diameter, $V_{C L}$ was the red blood cell centerline velocity and A was the cross-section area.

Mean arterial blood pressure (MABP; Viggo-Spectramed P10E2 transducer - Oxnard, CA, USA - connected to a catheter in the femoral artery) and heart rate were monitored with a Gould Windograf recorder (model 13-6615-10S, Gould, OH, USA). Data were recorded and stored in a computer. Blood gas measurements were carried out on arterial blood samples withdrawn from arterial catheter at $30 \mathrm{~min}$ time period intervals (ABL5; Radiometer, Copenhagen, Denmark). The hematocrit was measured under baseline conditions, at the end of BCCAO and at RE.

\section{WESTERN BLOT ANALYSIS}

Protein concentrations were determined by the Bio-Rad protein assay (Bio-Rad). To detect the proteins of interest, specific antibodies: anti-eNOS (mouse monoclonal antibody, 1:200, Santa Cruz Biotechnology, Santa Cruz, CA, USA), anti-phosphorylated eNOS [Rabbit (polyclonal) Anti-eNOS ( $\mathrm{pS}^{116}$ ) phosphospecific antibody unconjugated, Invitrogen] and anti- $\beta$-actin (mouse monoclonal, 1:1000, Sigma) were used. Immunoreaction was revealed using anti-mouse IgG conjugated to peroxidase, 1:2000 (GE Healthcare) by the ECL reagent (GE Healthcare). The optical density of the bands was determined by Chemi Doc Imaging System (Bio-Rad) and normalized to the optical density of $\beta$-actin. eNOS and phosphorylated eNOS were evaluated to define concentrations of the expressed and active proteins, respectively.

\section{STATISTICAL ANALYSIS}

All reported values are means $\pm S D$. Data were tested for normal distribution with the Kolmogorov-Smirnov test. Parametric (Student's $t$-tests, Anova, and Bonferroni post hoc test) or nonparametric tests (Wilcoxon, Mann-Whitney, and Kruskal-Wallis tests) were used; non-parametric tests were applied to compare diameter and length data among experimental groups. The statistical analysis was carried out by SPSS 14.0 statistical package. Statistical significance was set at $p<0.05$.

\section{RESULTS}

Pial arterioles were classified in five orders according to diameter, length, and branching as previously reported (Lapi et al., 2012).

\section{SHAM-OPERATED ANIMALS}

In sham-operated animals there were neither changes in arteriolar diameter nor increase in leakage (NGL: 0.02 \pm 0.01 ) nor adhesion of leukocytes $(1.0 \pm 0.5 / 100 \mu \mathrm{m}$ v.l./30 s) and perfused capillary length was $100 \pm 4 \%$ of baseline during the same period of observation as in the other experimental groups.

The animals treated with PKC inhibitor chelerythrine (3.0 mg/Kg b.w.) or TK inhibitor tyrphostin 47 (2.2 mg/Kg b.w.) did not show significant changes in all parameters when compared with baseline (Table 1).

Pilot studies showed i.v. administration of chelerythrine ( $3.0 \mathrm{mg} / \mathrm{Kg}$ b.w.) abolished the arteriolar responses due to topical application of Phorbol-12,13-dibutyrate (PDBu), a PKC activator, at the dosage $100 \mu \mathrm{M}$ ( $n=7$; Hudetz et al., 1995). Under control conditions, $\mathrm{PDBu}$ was effective in inducing transient arteriolar dilation by $12.0 \pm 2.0 \%$ of baseline in sham-operated animals within $10 \pm 1 \mathrm{~min}$ of administration, followed by a significant decrease in arteriolar diameter by $10.0 \pm 1.5 \%$ of baseline within $15 \pm 1 \min (n=7)$.

Tyrphostin 47 (2.2 mg/Kg b.w.) infusion completely suppressed order 2 arterioles dilation due to topical application of vascular endothelial growth factor (VEGF), a TK activator at the dosage $0.1 \mathrm{nM}$ ( $n=7$; Morii et al., 1986). The diameter increased by $16.0 \pm 2.5 \%$ of baseline in sham-operated animals treated with VEGF, $n=7$. MABP did not undergo with any significant variation.

\section{BCCAO-REPERFUSION}

At the end of BCCAO, ischemic animals (group I) showed a decrease in diameter of order 2 vessels by $19.0 \pm 3.0 \%$ of baseline (Figure 1A), with increased permeability (NGL: $0.21 \pm 0.03$, $p<0.01$ compared with $\mathrm{S}$ group) as previously reported (Lapi et al., 2012).

At the end of reperfusion, order 2 arterioles constricted by $14.5 \pm 3.3 \%$ of baseline (Figure 1A), venular permeability significantly increased (NGL: $0.47 \pm 0.04, p<0.01$ vs. S group), with marked leukocyte adhesion $(9.0 \pm 2.0 / 100 \mu \mathrm{m}$ v.l./30 s; $p<0.01$ vs. $S$ group) in venules and decreased capillary perfusion (PCL; $60 \pm 7 \%$ of baseline, $p<0.01$ vs. S group; Tables 1 and 2). In single pial venules (SPV, diameter $30-40 \mu \mathrm{m}$ ) the blood flow was reduced by $30 \pm 1 \%$ of baseline $(p<0.01$ vs. baseline: $247 \pm 10 \mathrm{nl} / \mathrm{s})$.

\section{QUERCETIN}

At the end of BCCAO quercetin highest dose caused ( $\mathrm{Q}_{3}$ group) an increase in order 2 arteriole diameter by $13.4 \pm 3.4 \%$ of baseline $(p<0.01$ vs. ischemic animals; Figure 1A), while preventing microvascular permeability (NGL: $0.08 \pm 0.02, p<0.01$ vs. S and I groups).

At RE order 2 arterioles dilated by $40 \pm 4 \%$ of baseline $(p<0.01$ vs. ischemic animals; Figure 1A), while leakage of FD70 was prevented (NGL: $0.15 \pm 0.02, p<0.01$ vs. $S$ and I groups) as well as leukocyte adhesion $(3.0 \pm 1.0 / 100 \mu \mathrm{m}$ v.l./30 s; $p<0.01$ vs. $\mathrm{S}$ and I groups). Capillary perfusion was protected (PCL: $90 \pm 4 \%$ of baseline, $p<0.01$ vs. S and I groups; Tables 1 and 2). SPV blood flow was significantly increased by $50.0 \pm 0.9 \%$ of baseline: $235 \pm 12 \mathrm{nl} / \mathrm{s}, p<0.01 \mathrm{vs}$. baseline and I group.

\section{PKC INHIBITION PLUS QUERCETIN}

Chelerythrine, PKC inhibitor, administered before quercetin (group CQ), blunted quercetin-induced effects after BCCAO, because there was reduction in diameter of all pial arterioles as observed in ischemic animals at the end of BCCAO (Figure 1B), while MABP did not significantly change (Table 3 ). Leakage significantly increased (NGL: $0.19 \pm 0.02)$ compared to the animals treated with $5.0 \mathrm{mg} / \mathrm{Kg}$ b.w. of quercetin $\left(p<0.01\right.$ vs. $\mathrm{Q}_{3}$ group, $p=$ NS vs. I group).

At RE, all arterioles dilated: by $19.0 \pm 2.4 \%$ of baseline in order $2\left(p<0.01\right.$ compared with $\mathrm{I}$ and $\mathrm{Q}_{3}$ groups; Figure 1B) with 
Table 1 | Drug treatment (T): doses, route, and time of administration in the different experimental groups.

\begin{tabular}{|c|c|c|c|c|}
\hline Group & H/R plus $T$ & NO H/R plus T & Time & $N$ \\
\hline । & Saline solution $1.5 \mathrm{ml}$ i.v. & & Prior to $\mathrm{BCCAO}$, at Reperfusion beginning & 20 \\
\hline $\mathrm{Q}_{3}$ & Quercetin 5.0 mg/Kg b.w. i.v. & & Prior to $\mathrm{BCCAO}$, at $\mathrm{R}$ beginning & 9 \\
\hline $\mathrm{CO}$ & Quercetin $5.0 \mathrm{mg} / \mathrm{Kg}$ b.w. i.v & & Prior to $\mathrm{BCCAO}$, at $\mathrm{R}$ beginning & \\
\hline TQ & Tyrphostin 472.2 mg/Kg b.w. i.v. & & Prior to quercetin & 9 \\
\hline \multirow{2}{*}{ LCO } & Chelerythrine $3.0 \mathrm{mg} / \mathrm{Kg}$ b.w. i.v. & & Prior to quercetin & \\
\hline & Quercetin $5.0 \mathrm{mg} / \mathrm{Kg}$ b.w. i.v & & Prior to $\mathrm{BCCAO}$, at $\mathrm{R}$ beginning & \\
\hline \multirow[t]{3}{*}{ LTQ } & L-NAME 11.0 mg/Kg b.w. i.v. & & Prior to quercetin & 9 \\
\hline & Tyrphostin 472.2 mg/Kg b.w. i.v. & & Prior to quercetin & \\
\hline & Quercetin $5.0 \mathrm{mg} / \mathrm{Kg}$ b.w. i.v & & Prior to $\mathrm{BCCAO}$, at $\mathrm{R}$ beginning & \\
\hline GQ & Quercetin 5.0 mg/Kg b.w. i.v & & Prior to $\mathrm{BCCAO}$, at $\mathrm{R}$ beginning & \\
\hline $\mathrm{C}$ & Chelerythrine $3.0 \mathrm{mg} / \mathrm{Kg}$ b.w. i.v & & Prior to $\mathrm{BCCAO}$, at $\mathrm{R}$ beginning & 9 \\
\hline $\mathrm{T}$ & Tyrphostin 472.2 mg/Kg b.w. i.v. & & Prior to $\mathrm{BCCAO}$, at $\mathrm{R}$ beginning & 9 \\
\hline G & Glibenclamide $1.0 \mathrm{mg} / 100 \mathrm{~g}$ b.w. i.v. & & Prior to $\mathrm{BCCAO}$, at $\mathrm{R}$ beginning & 9 \\
\hline S & & Saline solution $1.5 \mathrm{ml}$ i.v. & Twice at 50 min interval & 13 \\
\hline SC & & Chelerythrine $3.0 \mathrm{mg} / \mathrm{Kg}$ b.w. i.v. & Twice at 50 min interval & 5 \\
\hline ST & & Tyrphostin $472.2 \mathrm{mg} / \mathrm{Kg}$ b.w. i.v. & Twice at 50 min interval & 5 \\
\hline
\end{tabular}

H/R: animals subjected to hypoperfusion and reperfusion and NO H/R: animals not subjected to hypoperfusion and reperfusion; $N$, number of rats utilized.

Table 2 | Variations of the main parameters in sham-operated (S) group, ischemic (I) group, quercetin $\left(\mathrm{O}_{3}\right) \mathrm{group}(5 \mathrm{mg} / \mathrm{Kg} \mathrm{b.w.),} \mathrm{chelerythrine}$ plus quercetin (CQ) group, tyrphostin 47 plus quercetin (TQ) group, L-NAME and chelerythrine plus quercetin (LCQ) group, L-NAME and tyrphostin 47 plus quercetin (LTQ) group, chelerythrine and tyrphostin 47 plus quercetin (CTO) group, glibenclamide plus quercetin (GQ) group at the end of reperfusion.

\begin{tabular}{|c|c|c|c|c|}
\hline Group & Arteriolar diameter (\%) & $\begin{array}{l}\text { Microvascular } \\
\text { permeability (NGL) }\end{array}$ & $\begin{array}{l}\text { Leukocyte adhesion (number } \\
\text { of leukocytes } / 100 \mu \mathrm{m} \text { of } \\
\text { venular length } / 30 \mathrm{~s} \text { ) }\end{array}$ & Capillary perfusion (\%) \\
\hline । & $85.5 \pm 3.3^{\circ}$ & $0.47 \pm 0.04^{\circ}$ & $9.0 \pm 2.0^{\circ}$ & $60 \pm 7^{\circ}$ \\
\hline $\mathrm{O}_{3}$ & $140.0 \pm 4.0^{\circ *}$ & $0.15 \pm 0.02^{\circ *}$ & $3.0 \pm 1.0^{\circ} *$ & $90 \pm 4^{\circ *}$ \\
\hline $\mathrm{CO}$ & $119.0 \pm 2.4^{\circ * \#}$ & $0.40 \pm 0.05^{\circ \#}$ & $7.0 \pm 1.0^{\circ} \#$ & $62 \pm 7^{\circ \#}$ \\
\hline LCO & $93.5 \pm 3.0^{* \#}$ & $0.42 \pm 0.03^{\circ \#}$ & $7.0 \pm 1.0^{\circ \#}$ & $63 \pm 5^{\circ \#}$ \\
\hline LTQ & $91.0 \pm 2.0^{\circ * \#}$ & $0.37 \pm 0.03^{\circ * \#}$ & $5.0 \pm 1.0^{\circ} *$ & $80 \pm 6^{\circ * \#}$ \\
\hline сто & $89.5 \pm 1.0^{\circ * \#}$ & $0.42 \pm 0.03^{\circ \#}$ & $8.0 \pm 2.0^{\circ} \#$ & $60 \pm 4^{\circ \#}$ \\
\hline GQ & $99.0 \pm 4.0 * \#$ & $0.22 \pm 0.03^{\circ * \#}$ & $3.5 \pm 1.0^{\circ} *$ & $85 \pm 4^{\circ *}$ \\
\hline
\end{tabular}

Arteriolar diameter and capillary perfusion are reported as percent changes of $100 \%$ baseline values. NGL, normalized gray levels. ${ }^{\circ} p<0.01$ vs. $S$ group, ${ }^{*} p<0.01$ vs. I group, $" p<0.01$ vs. $Q_{3}$ group.

no significant changes in MABP (Table 3). Leakage was marked $\left(0.40 \pm 0.05, p<0.01\right.$ vs. $Q_{3}$ groups); the adherent leukocytes were $7.0 \pm 1.0 / 100 \mu \mathrm{m}$ v.l./30 s, as observed in ischemic animals ( $p=\mathrm{NS}$ vs. I group and $p<0.01$ vs. $\mathrm{Q}_{3}$ group); PCL significantly diminished compared with $\mathrm{Q}_{3}$ group $(62 \pm 7 \%$ of baseline, $p=\mathrm{NS}$ vs. I group, $p<0.01$ vs. $\mathrm{Q}_{3}$ group; Tables 1 and 2 ). SPV blood flow was significantly reduced by $25.7 \pm 0.8 \%$ of baseline: $215 \pm 11 \mathrm{nl} / \mathrm{s}$, $p<0.01$ vs. baseline, I and Q group.

\section{TK INHIBITION PLUS QUERCETIN}

Tyrphostin 47, TK inhibitor administration, before quercetin (group TQ) did not affect quercetin-induced dilation of arterioles: 
Table 3 | Mean arterial blood pressure (MABP) under baseline conditions, at the end of bilateral common carotid artery occlusion (BCCAO) and at the end of reperfusion (RE) in sham-operated (S) group, ischemic (I) group, quercetin $\left(\mathrm{O}_{3}\right)$ group $(5 \mathrm{mg} / \mathrm{Kg} \mathrm{b.w.),}$ chelerythrine plus quercetin (CO) group, tyrphostin 47 plus quercetin (TQ) group, L-NAME and chelerythrine plus quercetin (LCQ) group, L-NAME and tyrphostin 47 plus quercetin (LTQ) group, chelerythrine and tyrphostin 47 plus quercetin (СТQ) group, glibenclamide plus quercetin (GQ) group.

\begin{tabular}{lccc}
\hline Group & Baseline $(\mathbf{m m H g})$ & BCCAO $(\mathbf{m m H g})$ & RE $(\mathbf{m m H g})$ \\
\hline $\mathrm{I}$ & $101.0 \pm 3.5$ & $99.0 \pm 4.0$ & $100.0 \pm 3.5$ \\
$\mathrm{O}_{3}$ & $104.0 \pm 5.5$ & $102.0 \pm 4.0$ & $103.0 \pm 5.0$ \\
$\mathrm{CQ}$ & $99.0 \pm 5.0$ & $98.0 \pm 4.0$ & $100.0 \pm 4.5$ \\
$\mathrm{TQ}$ & $100.0 \pm 4.0$ & $99.0 \pm 5.0$ & $100.0 \pm 5.0$ \\
LCQ & $101.0 \pm 5.5$ & $99.0 \pm 5.0$ & $100.0 \pm 5.0$ \\
LTQ & $102.0 \pm 3.5$ & $100.0 \pm 4.5$ & $101.0 \pm 4.5$ \\
CTO & $101.0 \pm 5.0$ & $100.0 \pm 5.0$ & $101.5 \pm 4.0$ \\
GQ & $98.0 \pm 4.5$ & $96.0 \pm 4.0$ & $99.0 \pm 5.5$ \\
\hline
\end{tabular}

Values are means $\pm S D$.

by $5.0 \pm 2.7 \%$ of baseline in order 2 ( $p<0.01$ vs. controls) at the end of BCCAO (Figure 1B) without significant change in MABP (Table 3). Leakage was marked (NGL: $0.18 \pm 0.02$ ) as observed in ischemic animals ( $p=$ NS compared with I group, $p<0.01$ vs. $\mathrm{Q}_{3}$ group).

At RE tyrphostin 47 partially blunted only the dilation in smaller arterioles (increase in order 2 diameter by $20.7 \pm 2.5 \%$ of baseline, $p<0.01$ vs. I and $\mathrm{Q}_{3}$ groups; Figure 1B), while MABP did not significantly change (Table 3 ). There was increased leakage (NGL: $0.39 \pm 0.04$ ) compared to $\mathrm{Q}_{3}$ group ( $p<0.01$ vs. $\mathrm{Q}_{3}$ group), while leukocyte adhesion was partly prevented $(4.0 \pm 1.0 / 100 \mu \mathrm{m}$ v.l./30 s, $p<0.01$ vs. I and $\mathrm{Q}_{3}$ groups) and capillary perfusion was partially protected (PCL: $81 \pm 9 \%$ of baseline, $p<0.01$ vs. I group; Tables 1 and 2). In single pial venules blood flow decreased by $16.5 \pm 0.8 \%$ of baseline: $293 \pm 8 \mathrm{nl} / \mathrm{s}, p<0.01 \mathrm{vs}$. baseline, I and Q group.

\section{NO SYNTHASE AND PKC INHIBITION PLUS QUERCETIN}

At the end of BCCAO, L-NAME, eNOS inhibitor, administered before PKC inhibitor, chelerythrine, and quercetin (group LCQ) caused a decrease in diameter of all arterioles: by $8.2 \pm 2.0 \%$ of baseline in order $2(p<0.05$ vs. ischemic animals, $p<0.01$ vs. $\mathrm{Q}_{3}$ group; Figure 1C), without significant changes in MABP (Table 2). Permeability increased (NGL: $0.21 \pm 0.03, p=\mathrm{NS}$ vs. I group, $p<0.01$ vs. $\mathrm{Q}_{3}$ group), as reported in ischemic animals.

At RE, all arterioles constricted: by $6.5 \pm 3.0 \%$ of baseline in order 2 ( $p<0.01$ vs. $\mathrm{Q}_{3}$ group; Figure 1C), while no significant changes in MABP were detected (Table 3). Leakage was slightly different when compared with ischemic animals (NGL: $0.42 \pm 0.03$, $p=\mathrm{NS}$ vs. I group, $p<0.01$ vs. $\mathrm{Q}_{3}$ group). The other parameters did not significantly change compared with CQ group: adherent leukocytes were $7.0 \pm 1.0 / 100 \mu \mathrm{m}$ v.l./30 s, $p<0.01$ vs. $\mathrm{Q}_{3}$ group; $p=\mathrm{NS}$ vs. I group), while PCL was $63 \pm 5 \%$ of baseline $(p<0.01$ vs. $\mathrm{Q}_{3}$ group, $p=\mathrm{NS}$ vs. I group; Tables 1 and 2$)$. SPV blood flow decreased by $28.1 \pm 0.6 \%$ of baseline: $226.3 \pm 10.5 \mathrm{nl} / \mathrm{s}, p<0.01$ vs. baseline and $\mathrm{Q}$ group.

\section{NO SYNTHASE AND TK INHIBITION PLUS QUERCETIN}

At the end of BCCAO, L-NAME, eNOS inhibitor, prior to tyrphostin 47, TK inhibitor, and quercetin (group LTQ) caused decrease in diameter of all arterioles: by $9.0 \pm 3.0 \%$ of baseline in order 2 ( $p<0.01$ vs. $\mathrm{Q}_{3}$ group; Figure 1C). MABP did not significantly change (Table 3 ). Leakage was attenuated (NGL: $0.15 \pm 0.02, \mathrm{p}<0.01$ vs. I group) compared to ischemic animals.

At RE all arterioles constricted: by $9.0 \pm 2.0 \%$ of baseline in order 2 ( $\mathrm{p}<0.01$ vs. $\mathrm{Q}_{3}$ group; Figure 1C), without significant increase in MABP (Table 3). Leakage was not so high as in ischemic animals (NGL: $0.37 \pm 0.03, p<0.01$ vs. I group); adhesion of leukocytes was attenuated $(5.0 \pm 1.0 / 100 \mu \mathrm{m}$ v.l./30 s, $p<0.01$ vs. I group) and capillary perfusion was higher than in ischemic animals (PCL: $80 \pm 6 \%$ of baseline, $p<0.01$ vs. I and $\mathrm{Q}_{3}$ groups; Tables 1 and 2). SPV blood flow was reduced by $23.5 \pm 0.5 \%$ of baseline: $250 \pm 9 \mathrm{nl} / \mathrm{s}, p<0.01$ vs. baseline, I and $\mathrm{Q}$ group.

\section{PKC AND TK INHIBITION PLUS QUERCETIN}

At the end of BCCAO, the inhibition of both PKC and TK prior to quercetin (group CTQ) caused a decrease in diameter of all arterioles: by $12.0 \pm 1.5 \%$ of baseline in order 2 ( $p<0.01$ vs. $\mathrm{Q}_{3}$ group; Figure 1C); however, MABP did not significantly change (Table 3). Leakage was marked (NGL: $0.20 \pm 0.02, p=$ NS vs. I group, $p<0.01$ vs. $\mathrm{Q}_{3}$ group).

At RE the arterioles constricted: by $10.5 \pm 1.0 \%$ of baseline in order $2\left(p<0.01\right.$ vs. $Q_{3}$ group; Figure 1C), while MABP did not significantly change (Table 3 ). Leakage was pronounced (NGL: $0.42 \pm 0.03, p<0.01$ vs. $\mathrm{Q}_{3}$ group); the adherent leukocytes increased in number $(8.0 \pm 2.0 / 100 \mu \mathrm{m}$ v.l./30 s, $p<0.01$ vs. $\mathrm{Q}_{3}$ group), PCL diminished ( $60 \pm 4 \%$ of baseline, $p=\mathrm{NS}$ vs. I group, $p<0.01$ vs. $\mathrm{Q}_{3}$ group; Tables 1 and 2). SPV blood flow was markedly decreased by $31.8 \pm 0.7 \%$ of baseline: $237.3 \pm 9.5 \mathrm{nl} / \mathrm{s}$, $p<0.01$ vs. baseline and $\mathrm{Q}$ group.

\section{ATP-SENSITIVE POTASSIUM (KATP) CHANNEL INHIBITION PLUS OUERCETIN}

At the end of BCCAO $\mathrm{K}_{\mathrm{ATP}}$ channel inhibition prior to quercetin (group GQ) caused a slight decrease in diameter of all arterioles, by $5.4 \pm 2.1 \%$ of baseline in order $2\left(p<0.01\right.$ vs. I and $\mathrm{Q}_{3}$ groups), while microvascular permeability was not affected (NGL: $0.10 \pm 0.02, p<0.01$ vs. I group). MABP did not significantly change (Table 3 ).

At RE the arterioles did not significantly dilate compared with baseline ( $p<0.01$ vs. I and $Q_{3}$ groups), while MABP did not significantly change (Table 3 ). FD70 leakage was moderately prevented compared with that observed in $\mathrm{Q}_{3}$ group (NGL: $0.22 \pm 0.03$, $p<0.01$ vs. $\mathrm{S}, \mathrm{I}$, and $\mathrm{Q}_{3}$ groups). Adherent leukocytes were $3.5 \pm 1.0 / 100 \mu \mathrm{m}$ v.l. $/ 30 \mathrm{~s}$ ( $p<0.01$ vs. $\mathrm{S}$ and I groups). Capillary perfusion slightly decreased (PCL: $85 \pm 4 \%$ of baseline, $p<0.01$ vs. S and I groups; Tables 1 and 2). SPV blood flow was significantly reduced by $29.0 \pm 0.4 \%$ of baseline: $274 \pm 11 \mathrm{nl} / \mathrm{s}, p<0.01$ vs. baseline and Q group. 


\section{PKC, TK, AND K KATP CHANNEL INHIBITION PRIOR TO BCCAO AND REPERFUSION}

Protein kinase C inhibition by chelerythrine (C group) or TK inhibition by tyrphostin 47 ( $\mathrm{T}$ group) or $\mathrm{K}_{\mathrm{ATP}}$ channel inhibition by glibenclamide ( $\mathrm{G}$ group) did not affect the microvascular changes observed in rats submitted to BCCAO and reperfusion (Table 1).

Finally, physiological parameters, such as hematocrit, MABP, heart rate, $\mathrm{pH}, \mathrm{PCO}_{2}$, and $\mathrm{PO}_{2}$ did not change in the different experimental groups up to RE.

\section{eNOS EXPRESSION}

Western blot analysis showed that at the end of reperfusion eNOS protein concentration significantly increased in animals treated with highest dose quercetin compared with I and S group. On the contrary, there was no significant increase in animals treated with chelerythrine and tyrphostin 47 before quercetin when compared with I group (Figure 2). Moreover, eNOS protein concentrations were not uniformly expressed in cortex, striatum, and hippocampus, because the higher were detected in hippocampus and cortex, while the lower in striatum of all animals. The same trend was observed for phosphorylated eNOS protein concentrations: no significant increase after combined PKC and TK inhibition.

\section{DISCUSSION}

In rats submitted to hypoperfusion by BCCAO and reperfusion there were peculiar microvascular responses, characterized by decrease in arteriolar diameter, increase in microvascular permeability and leukocyte adhesion, reduction in capillary perfusion, as previously reported (Lapi et al., 2012).

Quercetin, a plant derived compound with multiple proposed therapeutic effects (Rice-Evans, 2001), was effective in reducing pial microvascular alterations caused by BCCAO and reperfusion, inducing dilation of arterioles, prevention of leakage and leukocyte adhesion, facilitating capillary perfusion. These protective effects have been observed to be partly reduced by inhibition of nitric oxide release by L-NAME, eNOS inhibitor, able to decrease dilation of arterioles and capillary perfusion, to increase leakage, while there were no significant effect on leukocyte adhesion (Lapi et al., 2012).

Previous data indicate quercetin is able to activate or inhibit intracellular signaling pathways (Williams et al., 2004), such as those triggered by PKC or TK; in particular, quercetin increases PKC activity derived from rat brain (Chiwororo and Ojewole, 2010). Moreover, PKC has been suggested to regulate eNOS expression and activity in vascular cells, through different isoform activation (Davda et al., 1994; Fleming et al., 2001; Michell et al., 2001). PKC- $\beta$ and PKC- $\varepsilon$ appear to inhibit NO release, while PKC- $\alpha$ and $\mathrm{PKC}-\delta$ are likely to increase NO formation in vessels (Partovian et al., 2005; Zhang et al., 2005; Motley et al., 2007; Fleming, 2008). Therefore, we hypothesized quercetin could activate different intracellular signaling pathways in our model. To define mechanisms involved in the quercetin-induced protective effects, PKC inhibition exerted by chelerythrine before quercetin was induced during BCCAO and reperfusion. The results indicate PKC inhibition significantly affected quercetin-triggered effects: the arterioles partially dilated at the end of reperfusion, while leakage and leukocyte adhesion were marked accompanied by capillary perfusion impairment. Therefore, our data suggest quercetin partially caused arteriolar dilation by activation of PKC intracellular signaling pathway promoting NO release. PKC appeared uniformly distributed in all pial arterioles, because there was homogenous response of all arteriolar orders after its inhibition. Furthermore, inhibition of both NO release and PKC signaling pathway abolished dilation induced by quercetin causing slight constriction of pial arterioles (by $6.5 \pm 3.0 \%$ of baseline) at the end of reperfusion and marked decrease in single pial venule blood flow (by $25.7 \pm 0.8 \%$ of baseline). Therefore, it is reasonable to suggest that the final result triggered by quercetin was mainly related to NO release, able to prevent blood-brain barrier disruption and to facilitate capillary perfusion.

Some in vitro studies indicate PKC activation can induce cerebral arteries constriction (Osol et al., 1991; Bonev et al., 1997). Our data show that PKC stimulation by PDBu induced dilation at first and then constriction of pial arterioles under baseline conditions. However, PKC inhibition by chelerythrine was not able to affect pial arteriolar diameter both in sham-operated animals and prior to BCCAO.

Quercetin is known to activate also the TK intracellular signaling pathway, as previously reported (Williams et al., 2004). In particular, TK has been reported to facilitate eNOS activation followed by increased NO release in arterioles under different conditions (Ayajiki et al., 1996; Corson et al., 1996; Fleming et al., 1998). Therefore, another hypothesis was TK inhibition could influence the quercetin-induced effects. Our data indicate TK inhibition by tyrphostin 47 administered prior to quercetin partly reduced dilation particularly in the smallest arterioles; this partial arteriolar dilation prevention might be due to decrease in NO release. The different arterioles responses to TK inhibition may be related to differentiated TK receptor distribution or involvement in dilation of pial arterioles. Consequently, the smaller ones were more responsive to tyrphostin 47 ; however, on the other end the decrease in single pial venule blood flow was by $16.5 \pm 0.8 \%$ of baseline, corresponding to attenuated leukocyte adhesion and capillary failure. Microvascular permeability increased when compared with that observed in quercetin-treated groups. Therefore, TK inhibition appears to affect more vessel permeability (higher compared to animals treated by quercetin alone) than other vascular parameters. Combined NO release and TK signaling pathway inhibition abolished quercetin-induced arteriolar dilation causing moderate constriction (by $9 \pm 2 \%$ of baseline) at the end of reperfusion corresponding to decrease in single pial venule blood flow by $23.5 \pm 0.5 \%$ of baseline. Therefore, TK pathway may partly contribute to the overall effects exerted by quercetin on pial microvasculature.

It is important to note PKC and TK intracellular signaling pathways were mainly triggered by quercetin administration, because PKC or TK inhibition did not cause any protective effects on pial microcirculation in animals not treated by quercetin and submitted to hypoperfusion and reperfusion.

It is worth underlying combined inhibition of PKC and TK pathways completely reversed protection triggered by quercetin causing the strongest arteriolar constriction (by $10.5 \pm 1.0 \%$ of baseline) at the end of reperfusion and marked decrease in single pial venule blood flow by $31.8 \pm 0.7 \%$ of baseline. These 
A
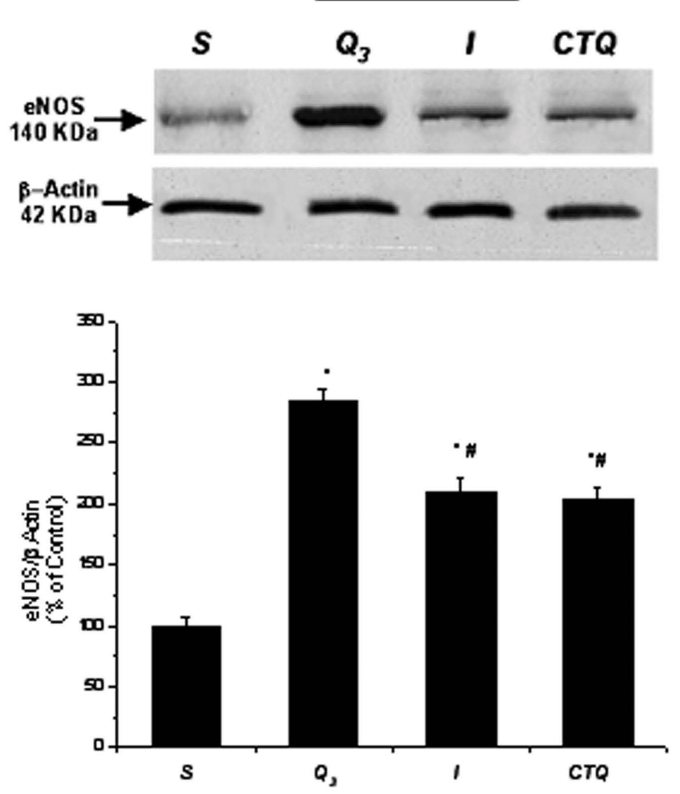

B

\section{STRIATUM}
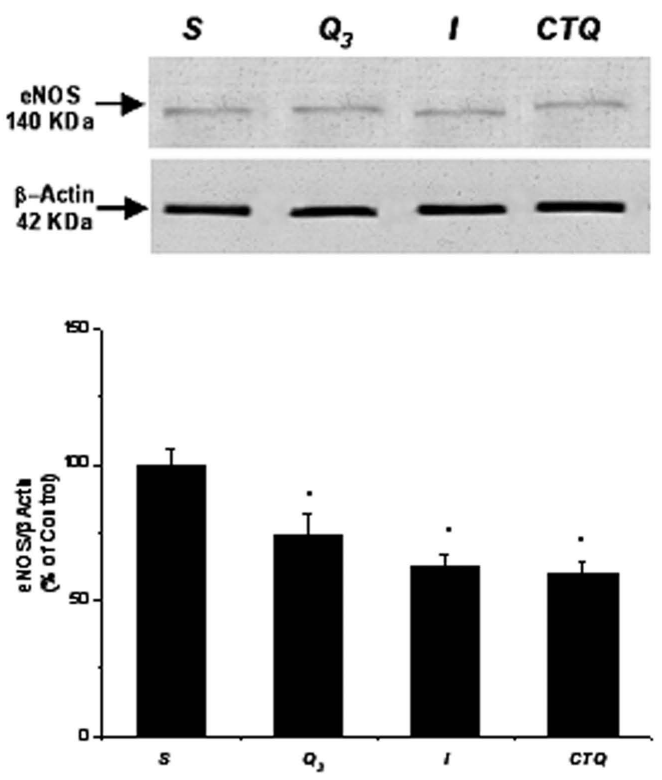

\section{c HIPPOCAMPUS}
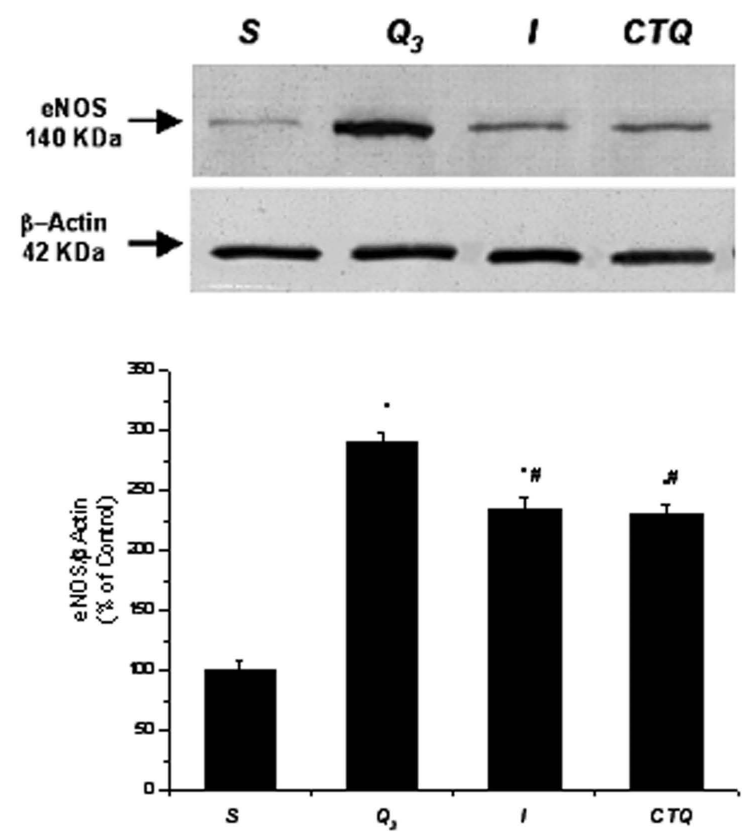

FIGURE 2 | Western blotting of eNOS expression in three cerebral zones [cortex (A), striatum (B), hippocampus (C)] at the end of reperfusion in sham-operated group ( $S$ group), in quercetin-treated (5 mg/Kg b.w.) group $\left(Q_{3}\right.$ group), in ischemic group (I group) and in chelerythrine and tyrphostin 47 plus quercetin-treated group (CTQ group). Corresponding densitometric values (mean $\pm S D$ ) are reported on the bottom. ${ }^{\circ} p<0.01$ vs. S group, $\# p<0.01$ vs. $\mathrm{Q}_{3}$ group effects indicate PKC and TK signaling pathways could mediate quercetin-induced pial vessel responses. In several previous studies quercetin has been observed to bind to the ATP-binding sites of a large number of proteins, such as PKC - modulating many cellular responses - such as inducible adhesion molecule ICAM1 expression in human endothelial cells (Kobuchi et al., 1999; 
Williams et al., 2004). Furthermore, quercetin may activate the MAPK pathway (ERK 2, JNK1, and p38) leading to expression of survival and defensive genes (i.e., glutathione- $S$-transferase, etc.) resulting in survival and protective mechanisms (homeostasis response; Kong et al., 2000). The effects on pial arteriolar dilation exerted by quercetin might be related to activation of $\mathrm{NO}$ releasing mechanisms through stimulation of both PKC and TK intracellular signaling pathways. Finally, both PKC and TK activation have been suggested to facilitate vascular preconditioning, i.e., coronary vasculature protection from endothelial dysfunction by ischemic preconditioning (brief heart exposure to ischemia before prolonged ischemia). These effects have been related to NO release through PKC and TK stimulation, as linear or parallel pathways (Sakamoto et al., 2005), and to $\mathrm{K}_{\mathrm{ATP}}$ channel (both mitochondrial and sarcoplasmatic) involvement in dilation mechanisms.

In our model, coupled inhibition of both $\mathrm{PKC}$ and TK pathways was able to abolish all effects exerted by quercetin, while inhibition of NO release did not completely suppress dilation. Western blot analysis, indeed, indicates that combined PKC and TK inhibition caused a decrease in eNOS expression and activation completely blunting quercetin's effects. Therefore, these effects on pial arteriolar dilation are mostly due to eNOS activation, although other mechanisms may contribute, such as $\mathrm{K}_{\mathrm{ATP}}$ channel involvement. To support this hypothesis we tested $\mathrm{K}_{\mathrm{ATP}}$ channel blocking effects

\section{REFERENCES}

Ayajiki, K., Kindermann, M., Hecker, M., Fleming, I., and Busse, R. (1996). Intracellular $\mathrm{pH}$ and tyrosine phosphorylation but not calcium determine shear stress-induced nitric oxide production in native endothelial cells. Circ. Res. 78, 750-758.

Bonev, A. D., Jaggar, J. H., Rubart, M., and Nelson, M. T. (1997). Activators of protein kinase $\mathrm{C}$ decrease $\mathrm{Ca}$ $2+$ spark frequency in smooth muscle cells from cerebral arteries. Am. J. Physiol. Cell Physiol. 273, C2090C2095.

Chau, C. H., Chen, K. Y., Deng, H. T., Kim, K. J., Hosoya, K., Terasaki, T., Shih, H. M., and Ann, D. K. (2002). Coordinating Etk/Bmx activation, and VEGF upregulation to promote cell survival, and proliferation. Oncogene 21, 8817-8829.

Chiwororo, W. D. H., and Ojewole, J. A. O. (2010). Dual effects of quercetin on rat isolated portal vein smooth muscle contractility. Cardiovasc. J. Afr. 21, 132-136.

Cho, J. Y., Kim, I. S., Jang, Y. H., Kim, A. R., and Lee, S. R. (2006). Protective effect of quercetin, a natural flavonoid against neuronal damage after transient global cerebral ischemia. Neurosci. Lett. 404, 330-335.

Cogolludo, A., Frazziano, G., Briones, A. M., Cobeno, L., Moreno, L., Lodi, F., Salaices, M., Tamargo, J., and Perez-Vizcaino, F. (2007). The dietary flavonoid quercetin activates BKCa currents in coronary arteries via production $\mathrm{H}_{2} \mathrm{O}_{2}$. Role in vasodilation. Cardiovasc. Res. 73, 424-431.

Colantuoni, A., Lapi, D., Paterni, M., and Marchiafava, P. L. (2005). Protective effects of insulin during ischemiareperfusion injury in hamster cheek pouch microcirculation. J. Vasc. Res. 42, 55-66.

Corson, M. A., James, N. L., Latta, S. E., Nerem, R. M., Berk, B. C., and Harrison, D. G. (1996). Phosphorylation of endothelial nitric oxide synthase in response to fluid shear stress. Circ. Res. 79, 984-991.

Davda, R. K., Chandler, L. J., and Guzman, N. J. (1994). Protein kinase $\mathrm{C}$ modulates receptor-independent activation of endothelial nitric oxide synthase. Eur. J. Pharmacol. 266, 237-244.

Fleming, I. (2008). "Biology of nitric oxide synthases," in Handbook of Physiology Microcirculation, eds R. F. Tuma, W. N. Duràn, and K. Ley (Amsterdam, NL: Academic Press), 56-80.

Fleming, I., Bauersachs, J., Fisslthaler, B., and Busse, R. (1998). Ca2+ -independent activation of the endothelial nitric oxide synthase in response to tyrosine phosphatase inhibitors and fluid shear stress. Circ. Res. 82, 686-695.

Fleming, I., Fisslthaler, B., Dimmeler, S., Kemp, B. E., and Busse, R. (2001). Phosphorylation of $\operatorname{Thr}(495)$

on arteriolar dilation triggered by quercetin and we observed suppression of dilation. Therefore, it is reasonable to suggest these channels contribute to arteriolar dilation and consequently to the overall effects induced by quercetin.

Furthermore, quercetin effects on leukocyte adhesion may be due to inhibition of adhesion molecule expression and quercetin anti-inflammatory properties. Therefore, quercetin's action appears to be complex, depending on several mechanisms including NO release stimulation. However, further studies are required to clarify all involved molecular mechanisms, in particular, the PKC isoforms role in the quercetin-induced protection.

In conclusion, quercetin prevented microvascular leakage and leukocyte adhesion accompanied by increase in arteriolar diameter and protection of capillary perfusion facilitating adequate blood flow as indicated by enhanced pial venular blood flow. Our data indicate quercetin's effects appear to be related to an increase in eNOS expression, NO release, and activity with the involvement, to a different extent, of PKC- and TK-dependent intracellular pathways. However, PKC and TK activation may also play a role in the prevention of blood-brain barrier disruption in association with quercetin scavenger activity, effective in preventing ROS formation and blood-brain barrier impairment (Fraser, 2011).

regulates $\mathrm{Ca}(2+) /$ calmodulindependent endothelial nitric oxide synthase activity. Circ. Res. 88, E68-E75.

Fraser, P. A. (2011). The role of free radical generation in increasing cerebrovascular permeability. Free Radic. Biol. Med. 51, 967-977.

Fryer, R. M., Schultz, J. E., Hsu, A. K., and Gross, G. J. (1999). Importance of PKC and tyrosine kinase in single or multiple cycles of preconditioning in rat hearts. Am. J. Physiol. 276, H1229-H1235.

Hudetz, A. G., Fehér, G., Weigle, C. G., Knuese, D. E., and Kampine, J. P. (1995). Video microscopy of cerebrocortical capillary flow: response to hypotension and intracranial hypertension. Am. J. Physiol. 268, H2202-H2210.

Huk, I., Nanobash, V. J., Weigel, G., Neumayer, C., Partyka, L., Patton, S., and Malinski, T. (1998). Bioflavonoid quercetin scavengers superoxide and increase nitric oxide concentration in ischemia-reperfusion injury: an experimental study. Br. J. Surg. 85, 1080-1085.

Kobuchi, H., Roy, S., Sen, C. K. Nguyen, H. G., and Packer, L. (1999). Quercetin inhibits inducible ICAM1 expression in human endothelial cells through the JNK pathway. Am. J. Physiol. 277, C403-C411.

Kong, A. N., Yu, R., Chen, C., Mandlekar, S., and Primiano, T. (2000). Signal transduction events elicited by natural products: role of MAPK and caspase pathways in homeostatic response and induction of apoptosis. Arch. Pharm. Res. 23, 1-16.

Lapi, D., Marchiafava, P. L., and Colantuoni, A. (2008). Pial microvascular responses to transient bilateral common carotid artery occlusion: effects of hypertonic glycerol. J. Vasc. Res. 45, 89-102.

Lapi, D., Vagnani, S., Pignataro, G., Esposito, E., Paterni, M., and Colantuoni, A. (2012). A. Protective effects of quercetin on rat pial microvascular changes during transient bilateral common carotid artery occlusion and reperfusion. Front. Physiol. 3:32. doi:10.3389/fphys.2012.00032

Michell, B. J., Chen, Z. P., Tiganis, T., Stapleton, D., Katsis, F., Power, D. A., Sim, A. T., and Kemp, B. E. (2001). Coordinated control of endothelial nitric-oxide synthase phosphorilation by protein kinase $\mathrm{C}$ and the cAMP-dependent protein kinase. J. Biol. Chem. 276, 17625-17628.

Moreau, P., Takasa, H., Küng, C. F., van Rooijen, M. M., Schaffner, T., and Lüscher, T. F. (1995). Structure and function of the rat basilar artery during chronic nitric oxide synthase inhibition. Stroke 26, 1922-1928.

Morii, S., Ngai, A. C., and Winn, R. (1986). Reactivity of rat pial arterioles and venules to adenosine and carbon dioxine: with detailed description of the closed cranial window technique in rats. J. Cereb. Blood Flow Metab. 6, 34-41. 
Motley, E. D., Eguchi, K., Patterson, M. M., Palmer, P. D., Suzuki, H., and Eguchi, S. (2007). Mechanism of endothelial nitric oxide synthase phosphorylation and activation by thrombin. Hypertension 49 , 577-583.

Negash, S., Gao, Y., Zhou, W., Liu, J., Chinta, S., and Raj, J. U. (2007). Regulation of cGMP-dependent protein kinase-mediated vasodilation by hypoxia-induced reactive species in ovine fetal pulmonary veins. Am. J. Physiol. Lung Cell Mol. Physiol. 293, L1012-L1020.

Ngai, A. C., Ko, K. R., Morii, S., and Winn, H. R. (1988). Effect of sciatic nerve stimulation on pial arterioles in rats. Am. J. Physiol. 254, H133-H139.

Oriji, G. K. (1999). Endothelin-induced prostacyclin production in rat aortic endothelial cells: role of calcium. Prostaglandins Leukot. Essent. Fatty Acid 61, 45-49.

Osol, G., Laher, I., and Cipolla, M. (1991). Protein kinase C modulates basal myogenic tone in resistance arteries from cerebral circulation. Circ. Res. 68, 359-367.

Pagliaro, P., Gattullo, D., Rastaldo, R., and Losano, G. (2001). Involvement of nitric oxide in ischemic preconditioning. Ital. Heart J. 2, 660-668.

Partovian, C., Zhuang, Z., Moodie, K., Lin, M., Ouchi, N., Sessa, W. C., Walsh, K., and Simons, M. (2005). PKC alpha activates eNOS and increases arterial blood flow in vivo. Circ. Res. 97, 482-487.

Picq, M., Dubois, M., Munari-Silem, Y., Prigent, A. F., and Pacheco, H. (1989). Flavonoid modulation of protein kinase $\mathrm{C}$ activation. Life Sci. 44, 1563-1571.

Rice-Evans, C. (2001). Flavonoid antioxidants. Cur. Med. Chem. 8, 797-807.

Sakamoto, K., Yonoki, Y., Kubota, Y., Kuwagata, M., Saito, M., Nakahara, T., and Ishii, K. (2005). Inducible nitric oxide synthase inhibitors abolished histological protection by late ischemic preconditioning in rat retina. Exp. Eye Res. 82, 512-518.

Williams, R. J., Spencer, J. P. E., and Rice-Evans, C. (2004). Flavonoids: antioxidants or signalling molecules? Free Radic. Biol. Med. 36, 838-849.

Xu, X. L., Feinstein, D. L., Santiza, R. A., Koenig, H. M., and Pellegrino, D. A. (2002). Agonist-specific differences in mechanisms mediatine eNOS-dependent pial arteriolar dilation in rats. Am. J. Physiol. 282, $\mathrm{H} 237-\mathrm{H} 243$.

Zhang, J., Baines, C. P., Zong, C., Cardwell, E. M., Wang, G., Vondriska, T. M., and Ping, P. (2005). Functional proteomic analysis of a three-tier PKC - Akt-eNOS signalling module in cardiac protection. Am. J. Physiol. Heart Circ. Physiol. 288, H954H961.

Conflict of Interest Statement: The authors declare that the research was conducted in the absence of any commercial or financial relationships that could be construed as a potential conflict of interest.

Received: 07 September 2011; accepted: 30 March 2012; published online: 27 April 2012.

Citation: Lapi D, Vagnani S, Pignataro $G$, Esposito $E$, Paterni $M$ and Colantuoni A (2012) Rat pial microvascular responses to transient bilateral common carotid artery occlusion and reperfusion: quercetin's mechanism of action. Front. Physio. 3:99. doi: 10.3389/fphys.2012.00099

This article was submitted to Frontiers in Vascular Physiology, a specialty of Frontiers in Physiology.

Copyright $\odot 2012$ Lapi, Vagnani, Pignataro, Esposito, Paterni and Colantuoni. This is an open-access article distributed under the terms of the Creative Commons Attribution Non Commercial License, which permits non-commercial use, distribution, and reproduction in other forums, provided the original authors and source are credited. 